'Departamento de Gestión y Políticas Públicas, Facultad de Administración y Economía, Universidad de Santiago. Santiago, Chile.

${ }^{2}$ Center for Expermental Social Science. Facultad de Administración y Economía.

Universidad de Santiago. Santiago, Chile.

${ }^{3}$ Escuela de Doctorado. Universidad de Girona. Girona, España.

${ }^{4}$ Department of Social Policy and Intervention, University of Oxford. Oxford, Inglaterra.

${ }^{5}$ Facultad de Psicología, Departamento de Psicología Social y Metodología de las Ciencias del Comportamiento,

Universidad del País Vasco.

San Sebastián, España.

asociólogo, Doctor en Derecho. bSociólogo. 'Socióloga, Phdc. dPsicólogo, Doctor en Psicología.

La investigación contó con el financiamiento del Instituto de Salud Pública de la Universidad Andrés Bello.

Recibido el 11 de diciembre de 2014, aceptado el 30 de junio de 2015.

Correspondencia a:

Juan Carlos Oyanedel Fanor Velasco 43, oficina F Santiago, Chile.

Teléfono: +56225823970

juan.oyanedel@usach.cl

\section{Validación del índice de bienestar personal (PWI) en usuarios vulnerables de servicios de salud en Santiago, Chile}

\author{
JUAN CARLOS OYANEDEL ${ }^{1,2, a}$, SALVADOR VARGAS ${ }^{2,3, b}$, \\ CAMILA MELLA ${ }^{2,4, c}$, DARÍO PÁEZ ${ }^{2,5, \mathrm{~d}}$
}

\section{Validation of the Personal Wellbeing Index (PWI) in vulnerable users of health care services in Santiago, Chile}

Background: Personal well-being calculates quality of life in terms of the necessary conditions required to live well. Aim: To validate the Personal Wellbeing Index (PWI) in a representative sample of vulnerable users of the public health system in Santiago, Chile. Material and Methods. A probabilistic and multistage sample consisting of 400 individuals aged $44 \pm 18$ years $(61 \%$ females) belonging to the lower income group of the National Health Fund (FONASA), residents of Gran Santiago was surveyed. Internal consistency and correlation between items and scale were examined. Structure was analyzed through confirmatory factor analysis. Results: The seven-item PWI is a good indicator of subjective well-being in the population under study, considering internal consistency, factor loadings, relation with overall life satisfaction and goodness of fit. The indicators mostly associated with personal well-being are the socioeconomic level followed by relationships with the community, health conditions and achievements. Conclusions: The 7-item version of the PWI is suitable for application in vulnerable health service users.

(Rev Med Chile 2015; 143: 1144-1151)

Key words: Personal satisfaction; Quality of life; Statistics; Validation.
1 1 debate, tanto en el ámbito de las políticas públicas, como en las ciencias sociales y de la salud ha incluido recientemente al bienestar subjetivo. Este corresponde a un concepto que comenzó a ser utilizado para medir el nivel de satisfacción de necesidades básicas de los individuos más allá de sus aspectos objetivos, tales como el nivel de cobertura de los servicios básicos ${ }^{1-3}$. En este sentido, Veenhoven ${ }^{4}$ señala que el bienestar subjetivo corresponde a un indicador que permite calcular la calidad de vida "realizada" en un país o categoría social, abordando la validación empírica de ideas acerca de las condiciones necesarias para vivir bien. La literatura sostiene que los países que presentan mayores niveles de bienestar subjetivo en sus poblaciones corresponden a aquellos que rescatan culturas más igualitarias, que enfatizan la autonomía individual, los valores post-materiales y el individualismo expresivo, caracterizándose por rasgos políticos más cercanos a la democracia y la tolerancia ${ }^{1,5-7}$.

En cuanto concepto, el bienestar subjetivo se refiere a las evaluaciones -tanto positivas como negativas- que las personas efectúan sobre su vida ${ }^{8,9}$. En este marco, es posible distinguir dos principales componentes ${ }^{10}$ : el primero corresponde al eudaimonismo, que refiere a la autorrealización de los individuos. El segundo componente es el hedónico, que corresponde al ámbito afectivo-emocional, el cual reconoce las emociones 
negativas y positivas. Es, precisamente, dentro de este componente que algunos autores identifican a la felicidad, en cuanto valoración afectiva global ${ }^{11}$. Dentro de la perspectiva hedónica, se incluye a la satisfacción vital, la cual remite al componente de carácter cognitivo, en relación al cual las personas evalúan cuán satisfechas se encuentran respecto a distintos aspectos de sus vidas -tales como su entorno, su desarrollo personal, su seguridad, entre otros ${ }^{12}-\mathrm{o}$ a su vida como un todo ${ }^{13}$. De este modo, existe consenso respecto a que el bienestar subjetivo corresponde al componente psicosocial de la calidad de vida ${ }^{14}$.

En este contexto, la literatura señala que el bienestar subjetivo es un detonante de la salud y la longevidad, logrando predecir la expectativa de vida, aun controlando por variables de riesgo clásicas, tales como el peso o la ingesta de alcohol, entre otras ${ }^{15}$. Por ejemplo, en el caso específico de los adultos mayores en Chile -grupo respecto del cual el estado de salud es un tema prioritario- en la medida en que mejor evalúan su nivel de salud, mejor es su evaluación respecto de su bienestar general $^{16,17}$.

Chile presenta altos niveles de bienestar subjetivo, resultados que replican la mayoría de los países, con excepción de aquéllos que enfrentan crisis sociales ${ }^{1}$. Por ejemplo, frente a la satisfacción global con la vida, el promedio en población infantil y adolescente es de 8,6, de acuerdo a una escala entre 0 y 10 puntos (donde 0 es completamente insatisfecho y 10 , completamente satisfecho $)^{18}$. Por su parte, la media en población adulta alcanza un puntaje de 7,2 en la misma escala ${ }^{19}$. Sin embargo, es importante precisar que existen diferencias según quintiles de ingreso: mientras el grupo de mayor poder adquisitivo presenta un promedio de 8,0 puntos, el más desfavorecido, sólo de $6,5^{19}$. Lo anterior confirma la asociación directa entre nivel socioeconómico y bienestar subjetivo, cuya correlación es cercana a $0,20^{1}$. En el caso de la población infantil, dichas diferencias también se presentan, sin embargo, las desigualdades socioeconómicas poseen un efecto mediador respecto al bienestar $^{20,21,22}$.

Antecedentes como los previos revisten una especial importancia en el caso de países como Chile, donde la expansión de servicios sociales -tales como salud y educación- se ha logrado a través de mecanismos de mercado. Ello ha derivado en el aumento de las coberturas sobre la base de una fuerte segmentación socioeconómica en la calidad de los servicios. Por ejemplo, en el caso del financiamiento de salud, destaca la reforma de 1981 que derivó en la creación del Fondo Nacional de Salud (FONASA) y de las Instituciones de Salud Previsional (ISAPRE) ${ }^{23}$. Así, los cotizantes se segmentan entre quienes pertenecen al sistema público (asociado a los sectores más vulnerables) y al sistema privado (vinculado a los sectores con mayor poder adquisitivo), respectivamente. Cabe señalar que sólo quienes pertenecen a FONASA, específicamente al grupo A o B, obtienen atención gratuita bajo la denominada modalidad de atención institucional ${ }^{24}$, al ser calificados como indigentes o contar con ingresos inferiores al salario mínimo ${ }^{25}$.

A partir de lo anterior, es posible sugerir que el desarrollo de medidas válidas y confiables del bienestar subjetivo se erige como un objetivo relevante en materia de investigación social y políticas públicas, considerando su impacto sobre ámbitos sanitarios y sus diferencias según nivel socioeconómico. Por consiguiente, el presente estudio buscó contribuir en esta dirección, a través de la validación del Índice de Bienestar Personal (PWI por su nombre en inglés) para la población de usuarios vulnerables de servicios de salud en el Gran Santiago, Chile, mayores de 18 años, considerando las particularidades de estos sectores carenciados.

\section{Material y Método}

Los datos que se presentan fueron desarrollados en el contexto de la Encuesta de Evaluación de Servicios de Salud de Usuarios de Fondo Nacional de Salud grupo A, encargada por el Instituto de Salud Pública de la Universidad Andrés Bello al Centro de Estudios Cuantitativos de la Universidad de Santiago, aplicada entre los días 9 y 18 de abril de 2014.

\section{Población y muestra}

La población del estudio corresponde a beneficiarios de FONASA, específicamente del grupo A, en el Gran Santiago.

La muestra, de carácter probabilístico y trietápico (manzana, vivienda, persona), seleccionó manzanas de viviendas en las comunas de $\mathrm{La}$ Granja, San Ramón, Peñalolén, Macul, Quinta 
Normal, Cerro Navia, Lo Prado, Estación Central, Recoleta, Renca, Lo Barnechea, Huechuraba, Pedro Aguirre Cerda y La Florida, con el promedio de 2,86 manzanas por comuna. Se excluyeron comunas con alto nivel socioeconómico, considerando los datos provistos por el marco muestral del Instituto Nacional de Estadísticas, en la medida que las chances de hallar individuos usuarios de FONASA nivel A eran muy bajas. En cada manzana se seleccionaron 10 viviendas, siguiendo un muestreo por salto sistemático, partiendo por la esquina norponiente siguiendo la orientación de las manecillas de un reloj. Al interior de la vivien$\mathrm{da}$, se seleccionó aleatoriamente a un encuestado mayor de 18, previa constatación verbal de que fuese usuario de FONASA nivel A.

La muestra posee un error asociado de $\pm 4,9 \%$, con un nivel de confianza de $95 \%$. La muestra consideró a 400 casos, en la que $61 \%$ corresponde a mujeres, siendo la edad promedio 44,4 años $(\mathrm{DE}=17,89)$.

\section{Instrumento}

El PWI fue desarrollado por The International Wellbeing Group, con el objetivo de medir bienestar subjetivo de acuerdo a siete dimensiones (correspondiendo un ítem a cada una), que derivan directamente de la pregunta por la satisfacción vital global, a saber: nivel de vida, nivel de salud, logros, relaciones personales, seguridad personal, comunidad y seguridad futura ${ }^{26}$, siendo estas las utilizadas en la validación del presente artículo. Adicionalmente, en su versión de ocho ítems se considera una dimensión asociada a la religión o espiritualidad, que alude a vivir conectado consigo mismo, al entorno y a lo sagrado ${ }^{27}$. Asimismo, en su versión de nueve ítems se incluye el indicador de satisfacción global con la vida (Overall Life Satisfaction Scale) que, utilizando la misma escala, pregunta a los encuestados "Pensando en su propia vida y en sus circunstancias personales, ¿cuán satisfecho está con su vida, como un todo?”

El instrumento posee una escala de respuesta tipo Likert, cuyo rango oscila entre 0 y 10 puntos, donde 0 representa a completamente insatisfecho y 10, completamente satisfecho. Para el cálculo del indicador final se obtienen los promedios generales por ítem y a nivel general.

Este instrumento ha sido, también, aplicado y validado en adolescentes y estudiante ${ }^{11,13,28,29,30}$, demostrando su equivalencia con el utilizado para la población general ${ }^{31}$ y en población con discapacidad intelectual ${ }^{32}$. En Chile, el PWI ha sido validado en jóvenes, demostrándose propiedades psicométricas satisfactorias ${ }^{18,33}$.

\section{Procedimiento}

El cuestionario fue aplicado en las viviendas de las personas seleccionadas, por parte de un encuestador perteneciente al equipo de trabajo del Centro de Estudios Cuantitativos de la Universidad de Santiago, quienes en su mayoría poseen experiencia en aplicación de instrumentos.

Los encuestados dieron su asentimiento informado, previa explicación de los objetivos, características y alcances del estudio, enfatizando los encuestadores que la participación era voluntaria, que podían negarse a responder aquellas preguntas que no deseasen hacerlo y que podían desistir de su participación en cualquier momento de la encuesta.

\section{Análisis de datos}

Para efectuar la validación del instrumento se llevó a cabo, en primera instancia, un análisis del comportamiento de las variables. A continuación se evaluó la consistencia interna, a través de alfa de Cronbach. A su vez, se calcularon las correlaciones de Pearson entre los ítems y la escala. Finalmente, se validó su estructura a través de un análisis factorial confirmatorio (AFC), integrando el indicador de satisfacción global con la vida (OLS), utilizando el estimador de máxima verosimilitud con errores robustos estandarizados (MLR), el cual se adecúa a distribuciones no normales ${ }^{34}$, como la que presentan los datos en el estudio, tal como se presentará en los resultados. En el caso de este último procedimiento, para evaluar el modelo se observan las cargas factoriales, las que deben ser superiores a 0,3 ; el coeficiente estructural de la predicción de la satisfacción global con la vida y el porcentaje de varianza explicado; y los estadísticos de ajuste índice comparativo de Bentler-Bonett (CFI) y el índice de Tucker Lewis (TLI), los que deben ser superiores a 0,9 , y la raíz del error cuadrático medio de aproximación (RMSEA) y la raíz estandarizada del error medio cuadrático (SRMR), los que deben ser inferiores a $0,08^{34}$.

El procesamiento estadístico de los datos fue realizado a través del software SPSS 21 , mientras que el análisis factorial confirmatorio se llevó a cabo mediante el programa Mplus 6.12. 


\section{Resultados}

En primer lugar, fue posible observar que todas las medias de los ítems superaron el valor de 6,5, siendo la más baja la relacionada con la seguridad futura, mientras que la más alta fue relaciones personales, seguida por los logros en la vida y espiritualidad.

El PWI en su versión de ocho ítems, en tanto, obtuvo un promedio de $7,19(\mathrm{DE}=1,51)$, mientras que con sólo 7 ítems; 8,14 ( $\mathrm{DE}=1,36)$.

La asimetría y la curtosis de las variables evidenciaron que los datos no se distribuyeron normalmente, con un nivel de confianza de $95 \%$, en la medida que al tipificar los coeficientes de las variables, se obtuvo que la asimetría superó el valor crítico de contraste de 1,96 en todas las variables, mientras que la curtosis en cuatro de ellas $^{35}$ (Tabla 1).

La consistencia interna del PWI con la totalidad de los ocho ítems obtuvo un valor de 0,82 , superando los valores mínimos aconsejados por la literatura que los sitúan en $0,70^{36}$. Todos los ítems de la escala se correlacionan directamente con el
PWI, de modo tal que es posible afirmar que se comportaron en el sentido esperado, ya que a mayor puntuación de estos, mayor satisfacción vital o bienestar subjetivo. Sin embargo, la dimensión asociada a espiritualidad tuvo una correlación baja $(r=0,27)$, lo que también se evidencia en que su eliminación aumenta la consistencia interna, situando a alfa de Cronbach en 0,85 (Tabla 2).

A partir de lo anterior, es posible señalar que para medir el bienestar subjetivo -a través del PWI- debería eliminarse el ítem relativo a la satisfacción con la espiritualidad o religiosidad para la población estudiada, debido a que, probablemente, remite a la dimensión más colectiva o social del bienestar subjetivo, tal como algunas investigaciones han demostrado ${ }^{37}$. Por consiguiente, se continuó el análisis con la versión de siete ítems.

En el AFC del PWI en su versión de 7 ítems (PWI-7) se evidenció que el indicador mayormente asociado a este es el nivel de vida. Esto puede comprenderse en la medida en que la población, compuesta por usuarios del sistema público de salud del grupo A, corresponde a un grupo de la

Tabla 1. Estadísticos descriptivos de los ítems del PWI-7

\begin{tabular}{|c|c|c|c|c|c|c|c|c|}
\hline & \multirow[t]{2}{*}{ Media } & \multirow{2}{*}{$\begin{array}{l}\text { Desv. } \\
\text { est. }\end{array}$} & \multicolumn{3}{|c|}{ Asimetría } & \multicolumn{3}{|c|}{ Curtosis } \\
\hline & & & $\begin{array}{l}\text { Esta- } \\
\text { dístico }\end{array}$ & $\begin{array}{l}\text { Error } \\
\text { típico }\end{array}$ & $\begin{array}{l}\text { Coef. } \\
\text { Tip. }\end{array}$ & $\begin{array}{l}\text { Esta- } \\
\text { dístico }\end{array}$ & $\begin{array}{l}\text { Error } \\
\text { típico }\end{array}$ & $\begin{array}{l}\text { Coef. } \\
\text { Tip. }\end{array}$ \\
\hline NV ¿Cuán satisfecho está con su nivel de vida? & 7,11 & 2,00 & $-0,29$ & 0,12 & $-2,34$ & 0,00 & 0,25 & $-0,01$ \\
\hline NS ¿Cuán satisfecho está con su nivel de salud? & 6,94 & 2,24 & $-0,39$ & 0,12 & $-3,16$ & $-0,18$ & 0,24 & $-0,72$ \\
\hline $\begin{array}{l}\text { LOG ¿Cuán satisfecho está con lo que está } \\
\text { logrando en su vida? }\end{array}$ & 7,43 & 2,07 & $-0,48$ & 0,12 & $-3,89$ & 0,01 & 0,25 & 0,04 \\
\hline $\begin{array}{l}\text { RP ¿Cuán satisfecho está con sus relaciones } \\
\text { personales? }\end{array}$ & 7,96 & 2,11 & $-1,05$ & 0,12 & $-8,44$ & 1,08 & 0,25 & 4,38 \\
\hline $\begin{array}{l}\text { SEG ¿Cuán satisfecho está con qué tan seguro } \\
\text { se siente? }\end{array}$ & 6,98 & 2,37 & $-0,60$ & 0,12 & $-4,87$ & 0,04 & 0,25 & 0,17 \\
\hline $\begin{array}{l}\text { COM ¿Cuán satisfecho está con sentirse parte } \\
\text { de su comunidad? }\end{array}$ & 6,93 & 2,55 & $-0,63$ & 0,12 & $-5,08$ & $-0,07$ & 0,25 & $-0,27$ \\
\hline $\begin{array}{l}\text { SEGF ¿Cuán satisfecho está con su seguridad } \\
\text { futura? }\end{array}$ & 6,88 & 2,14 & $-0,47$ & 0,13 & $-3,76$ & 0,20 & 0,25 & 0,78 \\
\hline $\begin{array}{l}\text { ESP ¿Cuán satisfecho está con su espiritualidad } \\
\text { o religiosidad? }\end{array}$ & 7,25 & 2,76 & $-0,96$ & 0,12 & $-7,67$ & 0,36 & 0,25 & 1,46 \\
\hline PWI-7 Índice de Bienestar Personal 7 ítems & 7,14 & 1,63 & $-0,57$ & 0,12 & $-4,69$ & 0,79 & 0,24 & 3,25 \\
\hline PWI-8 Índice de Bienestar Personal 8 ítems & 7,15 & 1,57 & $-0,60$ & 0,12 & $-4,91$ & 1,03 & 0,24 & 4,23 \\
\hline
\end{tabular}


Tabla 2. Consistencia interna del PWI-7

\begin{tabular}{|lcc|}
\hline & $\begin{array}{c}\text { Correlación de Pearson } \\
\text { elemento-total } \\
\text { Corregida }\end{array}$ & $\begin{array}{c}\text { Alfa de Cronbach } \\
\text { si se elimina } \\
\text { el elemento }\end{array}$ \\
\hline NV ¿Cuán satisfecho está con su nivel de vida? & 0,590 & 0,795 \\
\hline NS ¿Cuán satisfecho está con su nivel de salud? & 0,492 & 0,806 \\
\hline LOG ¿Cuán satisfecho está con lo que está logrando en su vida? & 0,693 & 0,781 \\
\hline RP ¿Cuán satisfecho está con sus relaciones personales? & 0,646 & 0,786 \\
\hline SEG ¿Cuán satisfecho está con qué tan seguro se siente? & 0,643 & 0,785 \\
\hline COM ¿Cuán satisfecho está con sentirse parte de su comunidad? & 0,515 & 0,805 \\
\hline SEGF ¿Cuán satisfecho está con su seguridad futura? & 0,597 & 0,793 \\
\hline ESP ¿Cuán satisfecho está con su espiritualidad o religiosidad? & 0,268 & 0,846 \\
\hline
\end{tabular}

población con carencias materiales importantes. Asimismo, otras dimensiones fuertemente asociadas fueron nivel de salud, logros y relaciones personales. Por otra parte, las relaciones con la comunidad obtienen un menor impacto en el índice. También es importante notar que existió relación entre logros y relaciones personales $(0,47)$; seguridad y relaciones con la comunidad $(0,56)$ y seguridad futura $(0,50)$; y relaciones con la comunidad con seguridad futura $(0,53)$.
La variable latente conformada por los siete ítems fue un eficaz predictor de la satisfacción con la vida en su conjunto (OLS), en la medida que el coeficiente estructural alcanzó a 0,88, explicando $77,1 \%$ de la varianza de este último indicador (Figura 1).

Cabe destacar que los índices de ajuste del modelo a los datos fueron aceptables y dentro de los rangos sugeridos por la literatura ${ }^{34}$, en tanto que el índice de ajuste comparativo de Bentler-Bonett

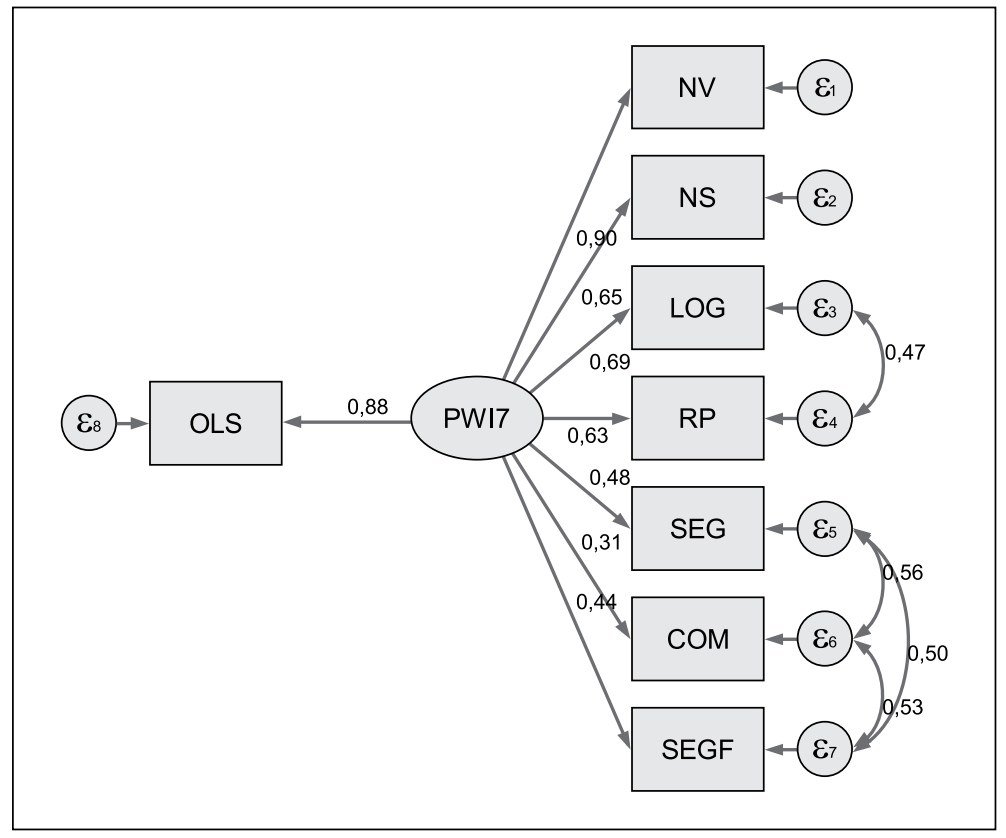

Figura 1. Análisis Factorial Confirmatorio del PWI-7. OLS: Satisfacción con la vida en su conjunto. PWI-7: Índice de Bienestar Personal de siete ítems. NV: Satisfacción con su nivel de vida. NS: Satisfacción con su estado de salud. LOG: Satisfacción con los logros alcanzados en su vida. RP: Satisfacción con las relaciones personales. SEG: Satisfacción con su seguridad. COM: Satisfacción con su comunidad. SEGF: Satisfacción con su seguridad futura. 
Validación del PWI en usuarios vulnerables de Servicios de Salud - J. C. Oyanedel et al

(CFI) fue 0,980, superior al criterio que este debe ser superior a 0,90; el índice de Tucker Lewis (TLI), 0,965 ; la raíz del error cuadrático medio de aproximación 0,048 ( $\mathrm{IC}=0,0210,074, \mathrm{p}>0,05)$; la raíz estandarizada del error medio cuadrático 0,050 .

\section{Discusión}

Los resultados que se presentan evidencian que el PWI, en su versión de siete ítems (PWI-7), se erige como un adecuado instrumento para medir bienestar subjetivo entre usuarios de sectores vulnerables y nivel socioeconómico bajo del sistema público de salud chileno (FONASA). Esto es posible de ser verificado por el hecho de que la variable latente que conforma el indicador es un predictor fuerte de la satisfacción global con la vida (OLS), el que es otro indicador de bienestar de amplio uso, al mismo tiempo que el ajuste del modelo fue adecuado utilizando cuatro estadísticos diferentes.

Cabe destacar que la configuración factorial del indicador, señala que la preocupación de esta población por la salud es secundaria, pero no pierde su relevancia, al situarse después del interés por el nivel de vida, junto con logros y relaciones personales. En efecto, este resultado es congruente con los hallazgos de estudios transculturales que muestran que la asociación entre la satisfacción con los ingresos y la satisfacción vital general es más fuerte en grupos de menores ingresos, pues las limitaciones del nivel de vida implican la dificultosa satisfacción de las necesidades básicas ${ }^{1}$. Lo anterior también se vincula al peso importante, pero menor, del nivel de salud ${ }^{19}$.

La satisfacción sobre las relaciones personales y la satisfacción con lo que se ha logrado en la vida poseen una asociación similar respecto de la satisfacción general. Ello es coherente con la asociación estable y de similar peso que han reportado los ámbitos como la familia y los amigos respecto de la satisfacción general en naciones de diferentes niveles de ingreso ${ }^{18}$. En efecto, es la importancia dada a las redes cercanas (interpersonales) en términos de integración, en detrimento a la valoración de la comunidad o de la sociedad, lo que también explicaría la baja asociación de la satisfacción con la comunidad y la satisfacción vital general, y de esta última con el bienestar espiritual.

Los resultados obtenidos permiten sugerir la construcción y aplicación de instrumentos como el PWI con indicadores que remitan a la evaluación de ámbitos específicos y diferenciados de la integración social, tales como las posibilidades de uso de tecnología o la vida sexual. Los hallazgos permiten también sugerir la construcción de indicadores vinculados con la medición de satisfacción para indagar en el componente subjetivo de los logros y avances en materia sanitaria, con el objetivo de determinar cómo perciben los usuarios los servicios de salud públicos, especificando sus distintos aspectos.

El estudio presentado posee limitaciones que deben ser consideradas con el objeto de evaluar adecuadamente sus resultados y conclusiones. La principal está asociada a la baja carga factorial de las dimensiones de sentimiento de formar parte de la comunidad y seguridad futura, las cuales, si bien cumplen con los valores mínimos absolutos de 0,3 sugeridos por la literatura, son menores al resto de las variables del indicador. Esto, en definitiva, enfatiza en la necesidad de corroborar los datos que se presentan en el estudio, con futuras investigaciones en la población de usuarios vulnerables de salud, con el fin de identificar si efectivamente son indicadores adecuados de bienestar.

\section{Referencias}

1. Diener E. Introduction The Science of Well-Being: Reviews and Theoretical Articles. En: Diener E, Editor, The Science of Well-Being. The Collected Works of Ed Diener. New York, Estados Unidos: Springer; 2009a. p. 1-10.

2. Diener E (Editor). Culture and Well-Being. The Collected Works of Ed Diener. New York, Estados Unidos: Springer; 2009b.

3. Oyanedel JC, Bilbao MA, Mella C. Midiendo el bienestar subjetivo: aspectos conceptuales y metodológicos. En: Martínez D, Ivanovic-Zuvic F, Unanue W, Editores, $\mathrm{La}$ Felicidad: Evidencias y Experiencias para Cambiar Nuestro Mundo. Santiago, Chile: LOM y Sociedad Chilena de Neurología, Psiquiatría y Neurología (SONEPSYN) 2013; p. 73-90.

4. Veenhoven R. El estudio de la satisfacción con la vida. Intervención Psicosocial 1994; 3 (9): 87-116.

5. Veenhoven R. Sociological theories of subjective wellbeing. En: Eid M, Larsen RJ, Editores, The Science of Subjective Well-being: A tribute to Ed Diener. New York, Estados Unidos: Guilford Publications; 2008. p. 44-61.

6. Delhey J, Dragolov G. Why inequality makes Europeans less happy: The role of distrust, status anxiety, and per- 
ceived conflict. Eur Sociol Rev 2014; 30 (2): 151-65 doi: 10.1093/esr/jct033.

7. Helliwell JF, Layard R, Sachs J (Editores). World Happiness Report. New York, Estados Unidos: Earth Institute, Columbia University; 2012.

8. Angner E. Subjective Well-Being. J Socio Econ 2010; 39 (3): 361-8. doi: 10.1016/j.socec.2009.12.001.

9. Diener E. Subjective well-being. En: Diener E, Editor, The Science of Well-Being. The Collected Works of Ed Diener. New York, Estados Unidos: Springer; 2009a. p. 11-58.

10. Vázquez C, Hervás G, Rahona JJ, Gómez D. Bienestar psicológico y salud: Aportaciones desde la Psicología Positiva. Anuario de Psicología Clínica y de la Salud 2009; 5: 15-28.

11. Casas F. Indicadores sociales subjetivos y bienestar en la infancia y adolescencia. En: Fondo de las Naciones Unidas para la Infancia, Editor, Propuesta de un Sistema de Indicadores sobre Bienestar Infantil en España. España: Editor; 2010. p. 34-51.

12. Oyanedel JC, Mella C. La felicidad como vocación o el bienestar como profesión: una mirada a la economía política del bienestar y la felicidad. En: Oyanedel JC, Mella C, Editores, Debates sobre el Bienestar y la Felicidad. Santiago, Chile: Ril; 2014. p. 211-23.

13. Casas F, Buxarrais MR, Figuer C, González M, Tey A, Noguera E, et al. Los valores y su influencia en la satisfacción vital de los adolescentes entre los 12 y los 16 años: estudio de algunos correlatos. Apuntes de Psicología 2004; 22 (1): 3-23.

14. Seligson JL, Huebner ES, Valois RF. Preliminary validation of the brief multidimensional students' life satisfaction scale (BMSLSS). Soc Indic Res 2003; 61 (2): 121-45. doi: 10.1023/A:1021326822957.

15. Diener E, Chan MY. Happy people live longer: Subjective well-being contributes to health and longevity. Appl Psychol Health Well Being 2011; 3 (1): 1-43. doi: 10.1111/j.1758-0854.2010.01045.x.

16. Alvarado X, Plaza A. Bienestar subjetivo, calidad de vida y envejecimiento en Chile. En: Oyanedel JC, Mella C, Editores, Debates sobre el Bienestar y la Felicidad. Santiago, Chile: Ril; 2014. p. 161-77.

17. Lacruz ME, Emeny RT, Baumert J, Ladwig KH. Prospective association between self-reported life satisfaction and mortality: Results from the MONICA/KORA Augsburg S3 survey cohort study. BMC Public Health 2011; 11 (1): artículo 579, 1-9. doi: 10.1186/1471-245811-579.

18. Oyanedel JC, Alfaro J, Varela J, Torres, J. ¿Qué Afecta el Bienestar Subjetivo y la Calidad de Vida de las Niñas y Niños Chilenos? Resultados de la Encuesta Internacional sobre Bienestar Subjetivo Infantil. Santiago, Chile: LOM; 2014.

19. Oyanedel JC, Browne M, Mella C, Ayala C. Epidemiología del bienestar subjetivo en Chile. En: Martínez D, Ivanovic-Zuvic F, Unanue W, Editores, La Felicidad: Evidencias y Experiencias para Cambiar Nuestro Mundo. Santiago, Chile: LOM y Sociedad Chilena de Neurología, Psiquiatría y Neurología (SONEPSYN); 2013. p. 53-72.

20. Oyanedel JC, Alfaro J, Mella C, Vargas S, Reyes F, Varela J. Children's well-being and socioeconomic inequality in Chile. Child Indic Res (en prensa).

21. Main G. Child poverty and children's subjective well-being. Child Indic Res 2014; 7 (3): 451-72. doi: 10.1007/ s12187-014-9237-7.

22. Cummins RA. Personal income and subjective well-being: A review. J Happiness Stud 2000; 1 (2): 133-58. doi: 10.1023/A:1010079728426.

23. Titelman D. Reformas al Sistema de Salud en Chile: Desafíos Pendientes (Serie Financiamiento del Desarrollo 104). Santiago, Chile: Comisión Económica para América Latina y el Caribe; 2000.

24. Fondo Nacional de Salud. Tramos ABCD. Disponible en: https://www.fonasa.cl/portal_fonasa/site/artic/20140621/pags/20140621192134.html [Consultado el 26 de junio de 2014].

25. Vergara-Iturriaga M, Martínez-Gutiérrez MS. Financiamiento del sistema de salud chileno. Salud Publica Mex 2006; 48 (6): 512-21.

26. The International Wellbeing Group. Personal Wellbeing Index-Adult (PWI-A). Manual 2013 (5 ed.). Melbourne, Australia: Australian Centre on Quality of Life, Deakin University; 2013.

27. Wills E. Spirituality and subjective well-being: Evidences for a new domain in the Personal Well-Being Index. J Happiness Stud 2009; 10 (1): 49-69. doi: 10.1007/ s10902-007-9061-6.

28. Tomyn AJ, Cummins RA. The subjective wellbeing of high-school students: Validating the Personal Wellbeing Index-School Children. Soc Indic Res 2011; 101 (3): 405-18. doi: 10.1007/s11205-010-9668-6.

29. Tomyn AJ, Norrish JM, Cummins RA. The subjective wellbeing of indigenous Australian adolescents: Validating the Personal Wellbeing Index-School Children. Soc Indic Res 2013; 110 (3): 1013-31. doi: 10.1007/s11205011-9970-y.

30. Casas F, Castellá J, Alfaro J, González M, Malo S, Bertran I, et al. Testing the Personal Wellbeing Index on 12-16 year-old adolescents in 3 different countries with 2 new items. Soc Indic Res 2012; 105 (3): 461-82. doi: 10.1007/s11205-011-9781-1. 
31. Tomyn A, Fuller M, Cummins R. The Personal Wellbeing Index: Psychometric equivalence for adults and school children. Soc Indic Res 2013; 110 (3): 913-24. doi: 10.1007/s11205-011-9964-9.

32. McGillivray JA, Lau ALD, Cummins RA, Davey G. The utility of the Personal Wellbeing Index Intellectual Disability Scale in an Australian sample. J Appl Res Intellect Disabil 2009; 22 (3): 276-86. doi: 10.1111/j.14683148.2008.00460.x.

33. Alfaro J, Valdenegro B, Oyarzún D. Análisis de propiedades psicométricas del Índice de Bienestar Personal en una muestra de adolescentes chilenos. Diversitas 2013; 9 (1): 13-27.
34. Wang J, Wang X. Structural Equation Modeling. Applications Using Mplus. New Jersey, Estados Unidos de América: Wiley; 2012.

35. Field A. Discovering statistics using SPSS (3a ed.). Los Angeles, Estados Unidos de América: Sage Publications; 2009.

36. Tabachnick BG, Fidell LS. Using Multivariate Statistics (5 ed.). Boston, Estados Unidos: Pearson; 2007.

37. Vargas S, Vargas F, Torres J, Bilbao M, García F, Oyanedel JC, et al. Bienestar y religión en Chile. En: Bilbao M, Oyanedel JC, Páez D, Editores, La felicidad de los chilenos. Estudios sobre bienestar. Volumen I. Santiago, Chile: Ril Editores; 2015. P. 151-65. 